\title{
Neisseria polysaccharea
}

National Cancer Institute

\section{Source}

National Cancer Institute. Neisseria polysaccharea. NCI Thesaurus. Code C86607.

A species of aerobic, Gram-negative, cocci shaped bacteria assigned to the phylum Proteobacteria. This species is catalase and oxidase positive, produces a yellowish pigment, reduces nitrate, but not nitrite, requires cystine-cysteine for growth, produces large amounts of polysaccharides, and produces acid from glucose and maltose, but not sucrose or lactose. N. polysaccharea is commensal in the nasopharynx of infants and children and is considered nonpathogenic. 